\title{
Generation of Complex Optical Pulse Sequences by Multiple Comb Shaping
}

\author{
Dmitry Pestov, Vadim V. Lozovoy and Marcos Dantus
}

O ptical pulse sequences, with controllable time delays and shapes, are an important class of waveforms used for autocorrelation and cross-correlation measurements, pump-probe and coherent control experiments, multidimensional nonlinear spectroscopy, etc. Arbitrary waveform generation requires the modulation of phase, amplitude and even polarization of the field. There has been a growing interest in simplifying pulse-train synthesis using limited degrees of freedom to control the spectral phase and amplitude of the field by shapers with a single-mask spatial light modulator (SLM). ${ }^{1-3}$

Here, we introduce an intuitive and versatile technique, called multiple independent comb shaping (MICS), which enables generation of complex optical pulse sequences via phase-only shaping of the input laser spectrum. ${ }^{4}$ Rather than trying to reconstruct the Fourier spectrum, which requires both phase and amplitude modulation, we interlace the spectral phase functions for each of the sub-pulses. For example, the technique allows us to obtain non-backgroundfree and interferometric autocorrelation traces without splitting and overlapping beams. It also enables straightforward and independent manipulation of the spectral phase for every sub-pulse in the programmed waveform. A pulse train consisting of three differently shaped pulses is easily obtained using phase-only MICS. The interferometric cross-correlation of such a pulse train is obtained by generating an additional fourth transformlimited pulse, which is scanned in time while the second harmonic generation signal is recorded. The time delay tuning is accomplished through pulse shaping by changing the slope of the linear phase function for the fourth pulse.

To summarize, MICS allows the straightforward design of pulse trains

(a)
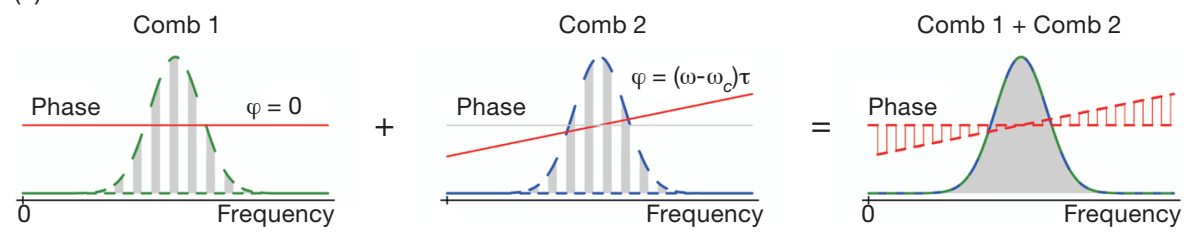

(b)

(c)
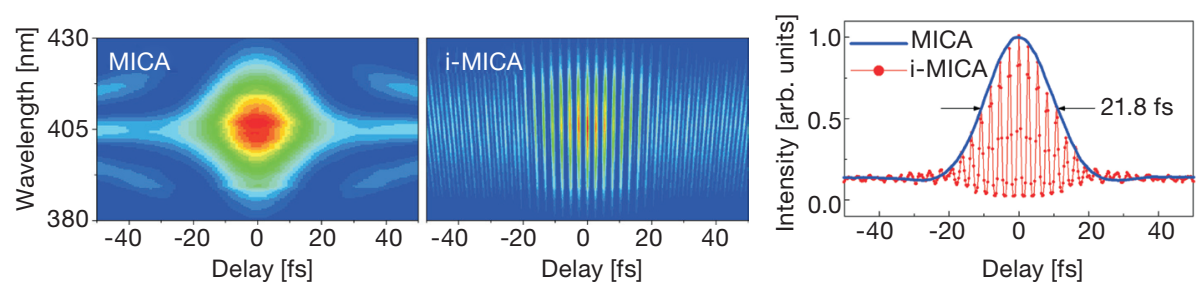

(d)

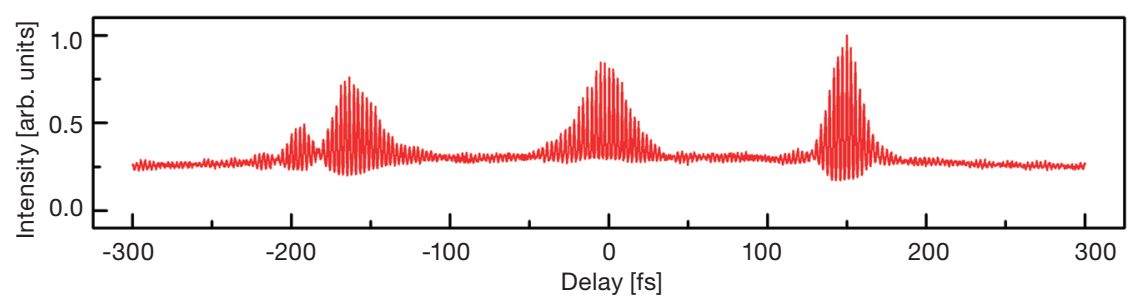

(a) Multiple Independent Comb Shaping (MICS) concept applied to the generation of a pair of delayed optical pulses. The two pulses, one at zero delay and the other at delay $\tau$, are created by encoding a piecewise phase mask across the spectrum. This phase is an alternating superposition of continuous phase functions applied to two independent combs of the available spectrum. Intensity and interferometric autocorrelation spectrograms. (c) Spectrally integrated intensity and interferometric autocorrelations. (d) Complex waveform generation: a $3+1$ pulse sequence, with the first pulse having third-order dispersion of $-5000 \mathrm{fs}^{3}$, the second with second-order dispersion of $+200 \mathrm{fs}^{2}$, and the third transform limited (TL). The fourth $\mathrm{TL}$ pulse is scanned to produce a cross-correlation trace. The data shown in (b-d) were acquired using a Ti:sapphire femtosecond oscillator, producing 15-fs pulses (central wavelength, 810 $\mathrm{nm}$ ), and a $4 \mathrm{f}$-shaper with a one-dimensional single-mask 640-pixel liquid-crystal SLM. Phase distortions in the original pulse were eliminated using MIIPS. ${ }^{5}$

for which the time delay, spectral phase function and relative phase are determined, even using a shaper with a single-mask, one-dimensional SLM. This last point is especially valuable for amplified laser systems, when the pulse shaper is placed before a regenerative amplifier and therefore amplitude modulation of the seeded spectrum is undesirable. $\Delta$

Dmitry Pestov, Vadim V. Lozovoy and Marcos Dantus (dantus@msu.edu) are with the department of chemistry, Michigan State University, East Lansing, Mich., U.S.A.

References

1. A. M. Weiner et al. J. Opt. Soc. Am. A 10, 1112-20 (1993).

2. J. C. Vaughan et al. Opt. Lett. 30, 323-5 (2005).

3. E. Frumker and Y. Silberberg. J. Opt. Soc. Am. B 24, 2940-7 (2007).

4. D. Pestov et al. Opt. Express 17, 14351-61 (2009).

5. Y. Coello et al. J. Opt. Soc. Am. B-Opt. Phys. 25, A140A150 (2008). 